\title{
Décadrages Décadrages
}

cinéma, a travers champs Cinéma, à travers champs

Anna Sanders Films, cinéma et art contemporain

\section{Des stratégies artistiques du réemploi à l'outil de production Anna Sanders Films}

François Bovier

\section{OpenEdition}

\section{Journals}

Édition électronique

URL : http://journals.openedition.org/decadrages/509

DOI : $10.4000 /$ decadrages. 509

ISSN : 2297-5977

Éditeur

Association Décadrages

Édition imprimée

Date de publication : 10 octobre 2008

Pagination : 9-16

ISBN : 978-2-9700582-8-1

ISSN : 2235-7823

Référence électronique

François Bovier, «Des stratégies artistiques du réemploi à l'outil de production Anna Sanders Films », Décadrages [En ligne], 13 | 2008, mis en ligne le 10 octobre 2009, consulté le 20 avril 2019. URL : http:// journals.openedition.org/decadrages/509; DOI : 10.4000/decadrages.509 


\section{Des stratégies artistiques du réemploi à l'outil de production Anna Sanders Films}

\author{
par François Bovier
}

Anna Sanders Films est une structure de production, créée en août 1997, par les artistes Charles de Meaux, Pierre Huyghe et Philippe Parreno, bientôt rejoints par Dominique Gonzalez-Foerster, en étroite collaboration avec Xavier Douroux et Frank Gautherot, fondateurs du centre d'art contemporain Le Consortium (initié en 1977 à Dijon, par l'association Le Coin du Miroir). Précisons encore que Frank Gautherot est responsable d'un bureau de graphisme, tandis que Xavier Douroux dirige la maison d'édition Les presses du réel. On le voit, dès ses origines, la société Anna Sanders Films est étroitement liée à des champs d'activité qui ne sont pas à proprement parler cinématographiques: en l'occurrence, à la diffusion d'art contemporain pour une part, à la publication de livres pour l'autre. C'est à partir de cette mixité originale et originelle que se déploie la Sàrl Anna Sanders Films. Il faut encore noter que, contrairement à la logique industrielle des grands trusts de cinéma, les coûts de production des films Anna Sanders n'excèdent pas les budgets réduits du cinéma d'essai (plafonnant autour des 300000 euros): leur financement repose principalement sur des subsides privés ou provenant d'institutions liées à l'art contemporain, dans une moindre mesure sur les aides à la production cinématographique telles que la commission d'avance sur les recettes du CNC.

\section{Cinéma, à travers champs}

On aimerait, dans cette introduction au dossier d'une revue de "cinéma» qui se positionne "à travers champs", reposer à partir de l'exemple emblématique d'Anna Sanders Films la question de l'interaction entre le cinéma et l'art contemporain. Actuellement, une tendance à affirmer la spécificité d'un espace d'intermédiation entre ces deux pratiques voit le jour: Pascale Cassagnau propose ainsi de ressaisir ces échanges à travers la catégorie de "troisième cinéma» $\mathbf{1}$ (sur le mode de la double négation: «ni» du cinéma expérimental «ni» de l'art vidéo, «ni» des films d’art et
1 Voir Pascale Cassagnau, Future Amnesia - Enquêtes sur un troisième cinéma, Editions Isthme, Paris, 2007. 
2 Voir Octavia Getino et Fernando Solanas, "Vers un troisième cinéma ", Tricontinental, n 3 , 1969, repris dans CinémAction, n 101 (René Prédal, éd., "Fernando Solanas ou la rage de transformer le monde»), 2002, pp. 96-114. Le terme "troisième cinéma " étant déjà utilisé en un sens référencié, vis-à-vis d'un engagement déterminé, il me paraît inapproprié de le réactualiser dans un contexte artistique contemporain dont on perçoit mal l'activisme politique.

3 Sur les discours de la spécificité du langage cinématographique dans la France des années 1920, voir Laurent Guido, L'âge du rythme. Cinéma, musicalité et culture du corps dans les théories françaises des années 1910-1930, Payot, Lausanne, 2007.

4 Voir l'ouvrage consacré aux productions Anna Sanders: The In-Between. Anna Sanders Films, Forma/Les presses du réel, Newcastle/Dijon, 2003.

5 Daniel Birnbaum, Chronologie, Les presses du réel/JRP Ringier, Dijon/Zurich, 2007, traduit de l'anglais par Simon Baril, pp. 76-78. Dans L'ellipse (1998), Pierre Huyghe rejoue une séquence de L'Ami américain (Der amerikanische Freund, Wim Wenders, 1977): dans le film de Wenders, Bruno Ganz, dans un appartement à Paris, parle au téléphone; le plan suivant le montre dans un autre appartement, situé de l'autre côté de la Seine. Huyghe insère entre ces deux plans Bruno Ganz qui se dirige, près de vingt ans plus tard, d'un appartement à l'autre, exhibant cette ellipse spatiale qui n'est pas visible en tant que telle dans le film de Wenders. essai «ni» des installations) - catégorie, ne l'oublions pas, qui est de fait inextricablement liée à la mouvance politique du cinéma du tiers-monde ou, plus précisément, du tiers-cinéma des années 1960-1970². Afin d'éviter toute équivoque, précisons encore que l'on utilise ici le terme de "spécificité» dans une optique historiographique, en pensant plus particulièrement à son application dans le contexte du cinéma: c'est-à-dire en tant qu'affirmation d'une identité inaliénable qui procède paradoxalement par comparaison, différenciation et opposition avec d'autres moyens d'expression (les arts déjà constitués, en ce qui concerne le cinéma) ${ }^{3}$. Cette contradiction dans les termes (le "troisième cinéma" se constituant en un "champ spécifique») est bien explicitée par la notion d'entre-deux («in-between » $\mathbf{4}$ : entre le cinéma et l'art contemporain) qui marque l'ambivalence ou l'impureté de cette intermédiation : la gageure, pour les plasticiens contemporains, consiste à parasiter, contaminer, court-circuiter les règles de l'art à travers les contraintes et les conventions du cinéma. Ce parasitage, rappelons-le, définit déjà le medium cinématographique, celui-ci se caractérisant dès ses origines par son hybridité, sa pluricodicité et sa transversalité.

Quoi qu'il en soit, on assiste indéniablement, depuis les années 1990, à un mouvement d'assimilation du cinéma par l'art contemporain. Comme le note Daniel Birnbaum à partir de l'œuvre de Pierre Huyghe, les artistes mobilisent couramment non seulement les techniques, les motifs et les procédés formels issus du cinéma, mais encore ses modalités de production collective et industrielle:

«Comme beaucoup d'artistes qui ont émergé durant les années 1990 [...], Huyghe a examiné les aspects technologiques ainsi qu'idéologiques du cinéma en tant qu'exemple paradigmatique de la culture du spectacle d'aujourd'hui. [...] Il a utilisé des scénarios, des bandes originales et des sous-titres comme points de départ dans ses différents projets, tout en s'intéressant au doublage, à la traduction, au remake. [...] Récemment, les appropriations en sont arrivées à un point où l'on peut se demander quel sens cela a, qu'une forme artistique (l'installation vidéo, par exemple) en cannibalise une autre (le cinéma). Aucun doute, l'installation vidéo en multiprojection introduit de nouvelles formes de récit et de participation du spectateur. Tout de même, pourquoi piller le cinéma? Peut-être que la réponse est que la vidéo pose des questions au cinéma que le cinéma est incapable de se poser à lui-même. C'est exactement ce que fait L'ellipse, par exemple, quand Huyghe nous fait prendre conscience de la fonction de la coupure en insérant la distance et le temps réels pour se rendre d'un point de l'histoire à un autre.» $\mathbf{5}$ 

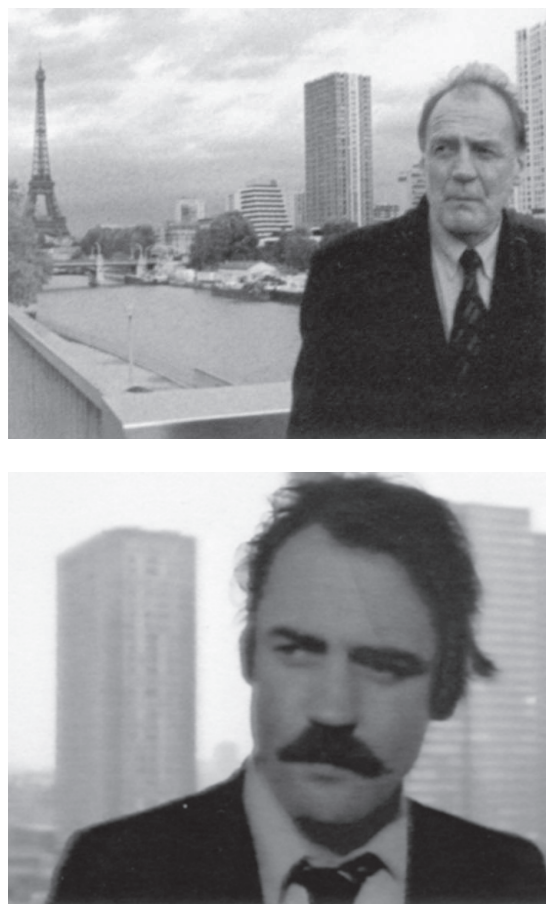

Der amerikanische Freund de Wim Wenders, 1977

Ce phénomène d'assimilation du cinéma par les artistes contemporains apparaît avec une rare évidence depuis le début des années 1990, mais il s'inscrit néanmoins dans une histoire déjà ancienne d'interaction entre ces deux champs de production culturelle, qui a connu différentes actualisations et configurations de sens. A cet égard, on peut sommairement distinguer deux principales modalités d'échanges, que l'on se propose de ressaisir à travers la catégorie du «film d'artistes»6, qui remonte aux années 1920, et celle du "cinéma de l'exposition» 7 , que l'on peut arbitrairement identifier à un phénomène relativement récent d'absorption du cinéma par les espaces d'art (c'est en effet le lieu de rappeler ici que le cinéma a été "exposé», sous une forme élargie, dès l'Exposition universelle de Paris, en 19008). Par ailleurs, comme le remarque Chris Darke ${ }^{9}$, le dispositif de la salle obscure lui-même peut être considéré comme une modalité d'exposition d'images en mouvement qui s'est normalisée et institutionnalisée avec le temps: le retour sur la «boîte noire» de la part d'un groupe d'artistes comme Anna Sanders permet d'interroger, de dénaturaliser et de déplacer la projection cinématographique et son espace public.
6 Certains historiens du cinéma, à l'instar de David Curtis en Grande-Bretagne, préfèrent la catégorie du "film d'artistes" à celle de "cinéma expérimental" (ou "visionnaire", "différent", "indépendant", "radical", " underground", etc.), les expérimentations filmiques étant souvent réalisées par des artistes (voir David Curtis, A History of Artists' Film and Video in Britain, British Film Institute, Londres, 2007). On peut noter que ce changement de terminologie est récent chez David Curtis, auteur par ailleurs d'un ouvrage devenu classique sur le cinéma des avant-gardes historiques et sur les films indépendants américains après la Seconde Guerre mondiale (Experimental Cinema, Delta, New York, 1971).

7 L'exposition d'images en mouvement n'est pas l'apanage des seules installations vidéo. Comme le souligne Dominique Païni, il y a également une histoire du cinéma exposé (voir Dominique Païni, Le temps exposé. Le cinéma de la salle au musée, Editions Cahiers du Cinéma, Paris, 2002), celui-ci étant théorisé dès les années 1970 à travers la catégorie de "cinéma élargi " (voir Gene Youngblood, Expanded Cinema, E. P. Dutton, New York, 1970).

8 Le cinéorama de Raoul Grimoin-Sanson (breveté en 1897), qui dispose une batterie de dix projecteurs synchronisés sur un écran circulaire, est présenté pendant trois jours à l'Exposition universelle de 1900. A cette même occasion, les frères Lumière projettent à la lumière du jour des films sur un écran de vingt mètres sur quinze mètres, visibles de part et d'autre de l'écran. Certes, il s'agit là de dispositifs attractionnels (étrangers au champ de l'art), mais ce sont ces mêmes dispositifs qui ont conduit dès les années 1970 les cinéastes expérimentaux et les artistes à faire retour sur le cinéma des premiers temps pour mettre en évidence sa dimension performative et ses affinités avec la fonction d'exposition.

9 Chris Darke, "Quelque part en marge du cinéma", The In-Between. Anna Sanders Films, op. cit., p. 58. 
10 Anna Sanders Films est une structure de production réunissant différents artistes, qui entend s'inscrire dans la logique économique et esthétique du cinéma d'auteur. Nous écartons donc comme points de référence ou antécédents directs les démarches d'artistes qui transposent à travers le format du cinéma des problématiques étroitement liées aux arts plastiques (l'on songe par exemple aux films de John Baldessari, Richard Serra, Robert Smithson), tout comme les entreprises individuelles d'artistes qui s'engagent dans l'industrie du cinéma, à l'instar de Robert Longo (Johny Mnemonic, E.-U., 1995) ou Cindy Sherman (Office Killer, E.-U., 1997).

11 La compagnie de production Zanzibar, fondée en 1968 par Sylvina Boissonnas en étroite relation avec Olivier Mosset, finance les films des cinéastes et artistes Serge Bard (Détruisez-vous, 1969), Patrick Deval (Acéphale, 1969), Philippe Garrel (La concentration, 1968; Le révélateur, 1968; Le lit de la vierge, 1969; La cicatrice intérieure, 1972), Daniel Pommereulle (Vite, 1969) et José Varela (Faire la déménageuse, 1972). Les films Zanzibar participent néanmoins à la culture cinéphilique française et aux courants libertaires issus de mai 1968, dont les artistes liés à Anna Sanders entendent se distancer. Sur les films Zanzibar, voir Sally Shafto, Zanzibar. Les films Zanzibar et les dandys de mai 1968, Editions Paris Expérimental, Paris, 2007.

12 Comme Peter Decherney l'a démontré, les films distribués par la Film-Makers' Cooperative reposent essentiellement sur le mécénat privé: Jonas Mekas, à travers différentes sociétés écran, sollicite régulièrement les fonds du mécène Jerôme Hill, le cinéma expérimental américain, contrairement à la peinture moderniste, n'étant pas parvenu à obtenir le soutien des musées, des fondations et des bourses d'artistes, des universités et du gouvernement. Voir Peter Decherney, Hollywood and the Culture Elite. How the Movies Became American, Columbia University Press, New York, 2005, pp. 161-203.

13 Evoquant des groupes de vidéastes qui ont vu le jour dans les années 1960-1970, tels que General Idea, Group Material ou encore Ant Farm, Pierre Huyghe oppose à ces structures politisées - qu'il décrit comme des "formats qui ne sont plus vraiment viables" aujourd'hui - des regroupements plus informels d'artistes, qu'il définit comme "des formes d'associations temporaires" qui reposent sur une réunion de singularités (Hans Ulrich Obrist, "Pierre Huyghe", Conversations. Vol. 1, Editions Manuella, Paris, 2008, p. 428).

\section{De l'activisme des collectifs d'artistes à la constitution d'un outil de production cinématographique}

Anna Sanders mobilise à la fois les stratégies du «film d'artistes» et celles du "cinéma de l'exposition", mais rarement en corrélation. Avant d'expliciter ce point, l'on entend souligner les divergences de positionnement entre cette structure de production et certains collectifs d'artistes qui se sont engagés antérieurement sur le terrain du cinéma ${ }^{\mathbf{1 0}}$.

En France, l'on pense évidemment au groupe Zanzibar11; mais à la différence d'Anna Sanders, le but est de financer, en toute indépendance, des projets de cinéastes ou d'artistes, dans la veine esthétique de ce que l'on pourrait désigner comme un «style international moderniste». Sur un plan plus structurel, on pourrait convoquer les différentes coopératives de cinéastes indépendants qui voient le jour à la suite de la Film-Makers' Cooperative de New York, que Jonas Mekas a fondée en 1962 (la Coopérative des cinéastes de Londres est créée en 1966, celle de Vienne en 1968, celle de Paris en 1974, etc.): mais en ce cas, les efforts se concentrent sur la diffusion, voire sur la conservation des films, et non sur leur production 12. Les films Fluxus, que George Maciunas réunit en une anthologie en 1970, paraissent constituer le précédent le plus évident (des artistes, réunis à travers un mouvement - de sensibilité néodadaïste - proposent une œuvre en commun : des Fluxfilms, comme ils ont pu produire différents objets et événements). Néanmoins, l'anthologie de Fluxfilms met en jeu des concepts artistiques qui ne croisent qu'épisodiquement l'histoire et les problématiques du cinéma (encore que la réunion de ces films en une anthologie par Maciunas peut évoquer les programmes composites des séances permanentes des années 1910); il n'y a guère que George Landow et Paul Sharits qui développent dans ce contexte un corpus cinématographique, qui est effectivement extrêmement proche d'interrogations relatives à l'art contemporain. Le support filmique, il faut en convenir, ne constitue plus un facteur de différenciation aujourd'hui (vis-à-vis du format de la vidéo). Et quand bien même admettrait-on cette distinction, comment alors considérer les films des artistes conceptuels Lawrence Weiner, David Lamelas ou encore Marcel Broodthaers, pour ne citer qu'eux? Si ces derniers ne sont pas issus de groupes ou de collectifs, ils prolongent néanmoins des démarches qui s'originent dans les arts visuels, ce qui n'est pas strictement ou uniquement le cas des productions Anna Sanders Films.

Les collectifs d'artistes vidéo des années 1960-1970, dont le programme est militant, ne constitue pas non plus un précédent direct. Comme Pierre Huyghe le souligne ${ }^{\mathbf{1 3}}$, sa démarche et par extension le projet Anna Sanders Films ne répondent pas à la dynamique politique 
et hiérarchique des "néo-avant-gardes». Selon nous, Anna Sanders se situe aux antipodes des collectifs militants des années 1960-1970. Si l'on sort du domaine de l'image animée, l'on pourrait convoquer comme modèle, qui est déplacé et inscrit dans un autre contexte, la critique institutionnelle de l'art et de ses instances de légitimation ${ }^{14}$ : dans ce cas, il s'agit de mettre en crise le statut de l'œuvre d'art, en inventant de nouvelles relations (le Département des Aigles, Musée d'art moderne que Marcel Broodthaers constitue dans son appartement en 1968, constitue peut-être l'emblème le plus marquant de cette tendance déconstructionniste). Anna Sanders, en un sens, joue le cinéma contre l'art: œuvre reproductible et collective, le film remet en cause un certain nombre de traits définitoires de l'art et des mécanismes de son marché. Mais entre la «critique institutionnelle de l'art» de la fin des années 1960 et la structure de production Anna Sanders, s'interpose un autre modèle: en l'occurrence, l'«esthétique relationnelle» de Nicolas Bourriaud (que ce dernier définit en ces termes: "théorie esthétique consistant à juger les œuvres d'art en fonction des relations interhumaines qu'elles figurent, produisent ou suscitent»15). L'œuvre, qui fait désormais "événement", se réduit aux échanges et aux interactions qu'elle instaure avec le public. Le cinéma, s'il se matérialise bien à travers la production d'objets filmiques ou vidéographiques, se déploie en un espace public mixte, "hétérotopique»16, qui excède les cercles restreints des spécialistes et des amateurs d'art contemporain : le film, tout en remettant en cause les frontières entre culture élevée et culture populaire, ne peut pas s'acquérir à l'instar d'une œuvre d'art signée et numérotée; produit de masse, il implique un espace ludique et une temporalité imposée au spectateur qui distingue ses conditions de réception de celles d'images en mouvement dans le cadre d'une exposition.

Lors d'un entretien avec Hans Ulrich Obrist, Dominique GonzalezFoerster revient sur le changement de paradigme qui est intervenu dans l'art contemporain à partir des années 1990, ce dernier se définissant désormais par des échanges entre pratiques culturelles (parmi lesquelles l'architecture, la musique, l'écriture) plutôt que par des collaborations entre artistes. Dans ce contexte, elle souligne la productivité du dispositif cinématographique qui permet de constituer une "zone d'articulation» ou d'opérer la "conjonction d'un vocabulaire», excédant ainsi l'exposition d'objets ready-made:

"Et c'est en ce sens que le cinéma, à mon avis, a servi de modèle. Si l'on s'est tellement servi de l'analogie avec le cinéma, c'est parce qu'on pouvait prendre exemple d'un langage qui articulait le son et l’image, qui reposait sur une conjonction d'éléments.»17
14 Sur ce point, voir par exemple Benjamin H. D. Buchloh, "De l'esthétique d'administration à la critique institutionnelle (aspects de l'art conceptuel, 1962-1969)", Essais historiques. Vol. 2, Art contemporain, Art Edition, Villeurbanne, 1992, traduit de l'anglais par Claude Gintz, pp. 155-212; Daniel Buren, "Fonction du musée" [1973], Les Ecrits (1965-1990), CAC Musée d'art contemporain, Bordeaux, 1991, pp. 195-204.

15 Nicolas Bourriaud, Esthétique relationnelle, Les presses du réel, Dijon, 1998, p. 117.

16 Sur la notion d'hétérotopie, voir Michel Foucault, "Des espaces autres" [1967], Dits et écrits. Vol. IV, 1980-1988, Gallimard, Paris, 1994, pp. 752-762. Miriam Hansen applique la notion comparable à plus d'un égard d'" espace public oppositionnel " (empruntée à Oskar Negt et Alexander Kluge, Öffentlichkeit und Erfahrung. Zur Organisationsanalyse von bürgerlicher und proletarischer Öffentlichkeit, Suhrkamp Verlag, Francfort-sur-le-Main, 1972) au cinéma pendant sa période d'institutionnalisation, soit entre les années 1907 et 1917. Voir Miriam Hansen, Babel and Babylon. Spectatorship in American Silent Film, Harvard University Press, Cambridge/Londres, 1991, pp. 90-125.

17 Hans Ulrich Obrist, "Dominique GonzalezFoerster", Conversations. Vol. 1, op. cit., p. 317. 
18 Charles de Meaux décrit Anna Sanders Films comme un "outil de production" qui "fonctionne comme une plate-forme mise au service des projets de chacun" des artistes impliqués dans cette structure (Hans Ulrich Obrist, "Charles de Meaux", Conversations. Vol. 1, op. cit., p. 554).

19 Deleuze et Guattari définissent en ces termes la structure du rhizome: "[...] à la différence des arbres ou de leurs racines, le rhizome connecte un point quelconque avec un autre point quelconque, et chacun de ses traits ne renvoie pas nécessairement à des traits de même nature, il met en jeu des régimes de signes très différents et même des états de non-signes. [...] II n'est pas fait d'unités, mais de dimensions, ou plutôt de directions mouvantes. II n'a pas de commencement ni de fin, mais toujours un milieu, par lequel il pousse et déborde. [...] Contre les systèmes centrés (même polycentrés), à communication hiérarchique et liaisons préétablies, le rhizome est un système acentré, non hiérarchique et non signifiant, sans Général, sans mémoire organisatrice ou automate central, uniquement défini par une circulation d'états." (Gilles Deleuze, Félix Guattari, Mille Plateaux. Capitalisme et schizophrénie, Editions de Minuit, Paris, 1980, pp. 31-32).

20 Ainsi, les films de Charles de Meaux ou d'Apichatpong Weerasethakul sont projetés aussi bien dans des festivals de cinéma, des salles spécialisées ou encore des ciné-clubs que dans des biennales d'art contemporain. Les installations de Pierre Huyghe ou de Philippe Parreno, par contre, sont montrées principalement dans des espaces d'exposition.

21 Citons en vrac quelques expositions monographiques organisées par Le Consortium depuis la fin des années 1970, pour donner la mesure de l'ampleur du travail effectué et son orientation anti-institutionnelle: Christian Boltanski, Annette Messager, Keith Sonnier, Cindy Sherman, Daniel Buren, Richard Prince, Carl Andre, Dara Birnbaum, Richard Serra, Bertrand Lavier, Hans Haacke, John Armleder, On Kawara, Steven Parrino, Olivier Mosset, Sylvie Fleury, Chris Burden, Philippe Parreno, Frank Stella, M \& M, Rirkrit Tiravanija, Donald Judd, Maurizio Cattelan, Pierre Huyghe, Angela Bulloch, Liam Gillick, Xavier Veilhan, Dominique Gonzalez-Foerster, Ange Leccia, Remy Zaugg, Carsten Höller, Amy O'Neill,...
Dans les faits, comme le producteur et réalisateur Charles de Meaux le souligne, Anna Sanders constitue un "outil de production»18. En ce sens, le modèle n'est plus celui de l'activisme politique et de la critique des media, mais celui du "rhizome» et des «inter-agencements" entre objets et subjectivités $\mathbf{1 9}$. On ne saurait dénier l'originalité de cette structure de production animée par un groupe d'artistes dont les écritures sont singulières, dissemblables. Ce qui peut par contre surprendre, c'est la relative absence de perméabilité entre les films et les installations produits par Anna Sanders. Les contextes de diffusion diffèrent; et si rapprochement il y a, c'est plutôt dans le sens de l'intégration des films au sein de l'espace muséal 20. En tout cas, la bipartition entre «films de cinéma» et "installations d'images en mouvement» n'est pas systématiquement transgressée: leur identité et leur différenciation génériques perdurent, même si certaines œuvres sont conçues pour transiter d'un format à l'autre. L'emprunt aux conventions du cinéma se situe peut-être sur un autre niveau, c'est-à-dire sur les modalités de production et de distribution des œuvres.

\section{L'appropriation du modèle de l'intégration verticale des studios par un groupe d'artistes}

Mon hypothèse de lecture est volontairement, résolument, provocatrice: je soutiendrai volontiers qu'Anna Sanders Films applique dans le champ de l'art, à travers un certain nombre d'écarts consentis et de distorsions créatives, la structuration verticale des grands studios de cinéma (entendre: un trust qui réunit les instances de production, de distribution et d'exploitation des films en une seule entité). L'art contemporain, depuis la fin des années 1960, procède régulièrement par appropriation, recyclage, emprunts et détournements. Les mass media, les codes de la publicité, les conventions du cinéma et des séries télévisées, pour renvoyer à quelques exemples patents, ont fait l'objet d'un travail de sape, de lecture critique ou encore de dénaturalisation. Le centre d'art du Consortium, auquel Anna Sanders se rattache historiquement, a exposé nombre d'artistes qui sont au centre de ces pratiques anti-culturelles (en ce sens qu'elles se portent à l'encontre des instances de légitimation de l'art) ${ }^{21}$. Combien même cette lecture constitueraitelle un coup de force (ou un forçage herméneutique, si l'on préfère), j'émets la gageure que l'"outil de production» Anna Sanders s'approprie avec succès les stratégies de structuration verticale des grands studios, dans un contexte économique qui n'est certes pas comparable. J'en veux pour preuve l'association d'Anna Sanders avec MK2: cette structure de diffusion de DVD fondée par Marin Karmitz, qui était à l'origine mili- 
tante, est devenue l'un des principaux supports de médiation du cinéma d'auteur en France; les films de Charles de Meaux ou d'Apichatpong Weerasethakul, les pièces de Dominique Gonzalez-Foerster, mais aussi les pochades (post)adolescentes et narcissiques de l'acteur Melvil Poupaud sont distribués par leur soin ; malgré la diversité des objets diffusés, la collection Anna Sanders acquiert une certaine homogonéité - par son institution en collection justement. La comparaison, il faut le concéder, atteint vite ses limites, Anna Sanders n'étant pas doté d'une filière d'exploitation. Il n'empêche, on assiste bien à une occupation du territoire du cinéma à travers une stratégie économique concertée: en se situant en marge de l'industrie du cinéma et en relation avec les instances du champ de l'art contemporain, Anna Sanders dessine un espace singulier qui est pourvu d'une certaine autonomie, les artistes-réalisateurs assumant la fonction de producteurs et de distributeurs ${ }^{22}$. Le caractère artisanal du cinéma expérimental est ainsi dépassé à travers l'instauration d'une société de production d'œuvres audio-visuelles, qui s'allie à un diffuseur de films d'art et essai.

Cet engagement dans la production cinématographique ne manque pas de poser la question des droits d'auteur et de la propriété intellectuelle. Blanche Neige Lucie (Pierre Huyghe, France, 1997), la première pièce produite par Anna Sanders Films (structure, rappelons-le, qui est fondée en vue de la réalisation du Pont du trieur de Charles de Meaux et Philippe Parreno ${ }^{23}$ ) inverse les termes de l'«expérience sociologique» intentée par Bertolt Brecht contre la Nero Film. Rappelons brièvement le différend qui oppose Brecht, et dans un premier temps Kurt Weill, à la Nero Film : le dramaturge et le compositeur contestent la violation du sens et de la portée politique de leur œuvre dans l'adaptation filmique de Georg W. Pabst (L'opéra de quat'sous, 1930); se heurtant à l'hégémonie de cette grande société de production, Brecht démontre, en perdant son procès, que l'industrie du cinéma exclut la notion même d'auteur, en réduisant l'œuvre à une marchandise $\mathbf{2 4}$. Par ce parallèle, l'on n'entend pas induire que Huyghe repart de Brecht, dans le procès que la doublure française de Blanche-Neige intente contre la société Disney, cette dernière ayant utilisé abusivement l'enregistrement de sa voix; mais le fait que Lucie Dolène obtienne compensation montre bien que tout a un prix (une valeur d'échange) dans la société administrée contemporaine, même une voix étrangère (et désolidarisée d'un corps qui est en luimême déjà artificiel, dessiné et animé image par image). Il s'agit dès lors, pour les artistes qui dirigent Anna Sanders, de jouer avec cette logique marchande, de la déplacer dans un contexte inapproprié (car en effet, comment la déjouer, lui échapper?), en la constituant en sujet d'une
22 Anna Sanders Films est une structure qui appartient de plein-pied au champ de la production cinématographique. Elle se distingue ainsi de l'entreprise de pointligneplan, collectif français qui se consacre à la programmation de films (cette structure est fondée en 1998 par Christian Merlhiot, rejoint l'année suivante par Pascale Cassagnau, Vincent Dieutre et Erik Bullot).

23 Sur ce point, voir dans le présent dossier l'entretien que Charles de Meaux nous a accordé.

24 "Quelle que soit la façon dont est conçue l'œuvre d'art et ce à quoi on l'a destinée, elle est désormais quelque chose qui se vend, et cette vente joue dans le système global des relations humaines un rôle dont l'importance est tout à fait nouvelle. [...] Si nous ne pouvons plus appliquer la notion d'œuvre d'art à la chose qui naît dès que l'œuvre d'art est transformée en marchandise, il faut alors abandonner cette notion, avec prudence et circonspection, mais sans crainte, si nous ne voulons pas que soit liquidée en même temps la fonction de la chose [...]. " (Bertolt Brecht, "Le procès de quat'sous" [1931], Sur le cinéma, L’Arche, Paris, 1970, traduit de I'allemand par Jean-Louis Lebrave et Jean-Pierre Lefebvre, pp. 214-215). 
25 En ouvrant le magazine édité par Huyghe et Parreno, on lit: "Anna Sanders est le nom d'un personnage de film en cours d'écriture. C'est aussi le nom d'un magazine qui présente ce personnage. Sans jamais voir aucune image de lui, on le découvre à travers le graphisme, les articles, le choix des images ou des publicités... D'autres personnages apparaîtront de la même manière, en donnant leur nom à d'autres numéros. L'ensemble de ces magazines racontera une histoire, incarnée par ces personnages que l'on ne voit jamais, donnant le sentiment d'une histoire, l'histoire d'un sentiment.." A la suite de l'éditorial, un avertissement est adressé au lecteur: "Anna Sanders n'est pas une personne que l'on peut apercevoir en couverture. Vous ne verrez aucune image d'elle, pourtant elle est omniprésente - dans le choix des articles, des photos et de la conception graphique."

26 Rappelons que Heidegger théorise à partir de la poésie de Hölderlin l'ouverture de "l'entredeux" (Zwischen) comme le mouvement définitoire de l'être-là (Dasein) et son mode d'habitat. Voir Martin Heidegger, "Hölderlin et l'essence de la poésie" [1936], Approches de Hölderlin, Gallimard, Paris, 1973, traduit de l'allemand par Henry Corbin, Michel Deguy et François Fédier, pp. 41-61. œuvre d'art: c'est bien là le projet auquel répond Anna Sanders Films, en s'adaptant aux régles de l'économie du cinéma.

\section{Anna Sanders Films : fictions, portraits, paysages}

Anna Sanders répond encore à un programme poétique qui peut se résumer à trois problématiques entrelacées. Anna Sanders, c'est d'abord un magazine édité par Pierre Huyghe et Philippe Parreno, en 199725 : un numéro unique est consacré à la création d'un personnage de fiction et à l'invention d'un scénario qui n'est pas destiné à être tourné. Cette virtualité d'une fable potentielle et d'un personnage imaginaire est au centre des films produits par la Sàrl Anna Sanders, neutralisant les indices qui permettent de distinguer les modes de la fictionnalité et de la documentarisation. A ce personnage de fiction, ou si l'on préfère à cette fiction (de) productrice d'images, se superposent deux projets ou intentions qui sont dé-différenciés: d'une part dresser le portrait d'un paysage comme l'on cerne les caractéristiques d'un personnage, d'autre part établir la légende d'un personnage comme cela se fait dans un atlas de géographie. En ce sens encore, la structure Anna Sanders est liminaire, incarnant le concept de l'«entre-deux»(Zwischen) ${ }^{\mathbf{2 6}}$, spécifié cette fois respectivement comme l'altérité du caractère ou de la personnalité, comme l'atopie ou l'exterritorialité au lieu. En effet, le film inaugural d'Anna Sanders: Le pont du trieur, est construit, en référence au mode d'énonciation des émissions radiophoniques, comme le relevé topographique d'un ailleurs, en l'occurrence la région du Pamir. Et le projet collectif AnnLee, produit par la société Anna Lena, consiste justement à doter d'une identité et d'une histoire un personnage désinvesti de manga, une "coquille vide» pour le dire succinctement. Le paysage fait histoire, et le personnage se déchiffre comme les légendes d'une carte topographique. Le binôme art contemporain et cinéma repose en ce cas sur un désir de fiction, suivant un mouvement d'effraction que l'on peut définir comme un délit de fabulation, une affabulation ou une contre-façon. 